\title{
Полевая диффузия в неупорядоченных органических материалах в условиях заполнения глубоких состояний
}

\author{
(C) В.Р. Никитенко, А.Ю. Кудров \\ Национальный исследовательский ядерный университет „МИФИ“, \\ 115409 Москва, Россия \\ E-mail: vladronik@yandex.ru
}

(Получена 16 мая 2016 г. Принята к печати 16 июня 2016 г.)

Развита простая аналитическая модель для коэффициента полевой диффузии при умеренной концентрации носителей заряда. Прыжковый транспорт описывается моделью многократного захвата на основе концепции транспортного уровня. Получено уравнение непрерывности с коэффициентом диффузии, зависящим от концентрации носителей, найдена зависимость коэффициента полевой диффузии от времени в нестационарных условиях. Получены оценки интервалов времени, на которых заполнение глубоких состояний влияет на подвижность и коэффициент диффузии в условиях времяпролетного эксперимента. Показано, что коэффициент полевой диффузии возрастает на длительном временно́м интервале, хотя подвижность постоянна, что напоминает случай неравновесной начальной генерации в предельном случае низкой концентрации.

DOI: 10.21883/FTP.2017.02.44098.8166

\section{1. Введение}

Органические полупроводники и диэлектрики применяются во многих электронных приборах - это светодиоды, фотовольтаические элементы, полевые транзисторы, устройства памяти. Перенос (транспорт) носителей заряда - это один из базовых физических процессов, определяющих характеристики электронных устройств. Транспорт является прыжковым [1,2], т.е. происходит путем некоррелированных туннельных перескоков между локализованными состояниями, энергии которых разбросаны в широких пределах (ширина распределения много больше тепловой энергии), закон сохранения энергии при переходах обеспечивается взаимодействием с фононами (колебаниями решетки). Подвижность в достаточно толстых слоях органических материалов, как и других высокоомных полупроводников, при не слишком малой толщине слоя (значительно превышающей глубину поглощения возбуждающего излучения) обычно измеряется по времяпролетной методике [1], которая предполагает генерацию избыточных носителей заряда (обычно импульсом ультрафиолетового света). Первоначально тонкий пакет фотогенерированных носителей заряда движется к противоположному электроду. Известно, что сигнал переходного тока, наблюдаемый в экспериментах по измерению времени пролета, имеет аномально широкий участок убывания при временах, превышающих время пролета, даже если до времени пролета наблюдается участок постоянства тока, так что постоянное значение подвижности успело установиться $[1,3]$. Во многих случаях, наблюдаемый сигнал может быть описан на основе уравнения непрерывности, которое включает не зависящие от времени подвижность и коэффициент диффузии, но последний оказывается много больше, чем следует из известного соотношения Эйнштейна [1-5]. Таким образом, дисперсия (разброс координат) дрейфующего пакета носителей заряда аномально велика, несмотря на отсутствие дисперсионного транспорта [1]. Это явление известно как полевая, или стимулированная полем диффузия (ПД). Его причина в статистическом разбросе времен освобождения носителей с глубоких состояний, прежде всего за счет разброса энергий состояний. Этот эффект был неоднократно описан теоретически $[4,6,7]$, но при условии низкой концентрации носителей, когда можно пренебречь заполнением глубоких состояний. В недавней работе [8] показано, что коэффициент ПД значительно убывает с ростом концентрации носителей. В данной работе, в согласии с результатом работы [8], получено простое аналитическое выражение для коэффициента ПД в зависимости от концентрации, а также его зависимость от времени в нестационарных условиях (например, времяпролетный эксперимент), когда концентрация зависит от времени и координат. Использованное приближение применимо при умеренных концентрациях, которые не влияют на величину подвижности, но достаточны для существенного убывания коэффициента ПД. Хотя в условиях времяпролетного эксперимента концентрация носителей заряда достаточно мала, чтобы электрическое поле в слое можно было считать постоянным и однородным, концентрация должна быть достаточно велика, чтобы обеспечить уровень сигнала, достаточно высокий для измерений.

\section{2. Теоретическая модель}

Для того чтобы получить выражение для коэффициента полевой диффузии, используется описание прыжкового транспорта в рамках модели многократного захвата (M3) [4], на основе концепции транспортного уровня [6]. Для определенности рассмотрим перенос электронов. Модель МЗ разработана и применяется для описания 
транспорта избыточных носителей в неупорядоченных полупроводниках с квазинепрерывным и достаточно широким (в сравнении с тепловой энергией) спектром локализованных состояний ниже дна зоны проводимости (с энергией $E_{c}$ ), таких как аморфный кремний, $\mathrm{As}_{2} \mathrm{Se}_{3}$ и др. Энергию $E_{c}$, разделяющую протяженные и локализованные состояния, называют также краем подвижности. Концентрация электронов, зависящая от координат (плоская геометрия) и времени, $p(x, t)$, складывается из концентрации подвижных (занимающих состояния с энергиями $\left.E \geq E_{c}\right)$ и локализованных $\left(E<E_{c}\right)$ носителей:

$$
p(x, t)=p_{c}(x, t)+\int_{-\infty}^{E_{c}} d E \rho(x, t, E) .
$$

Уравнение баланса для функции $\rho-$ энергетической плотности заполненных локализованных состояний (ловушек), $\rho(x, t, E)=g(E) f(x, t, E), g(E)$ - энергетическая плотность ловушек, $f(x, t, E)$ - вероятность их заполнения, записывается, вообще говоря, следующим образом:

$$
\begin{aligned}
\frac{\partial \rho(x, t, E)}{\partial t}= & C(E)[g(E)-\rho(x, t, E)] \rho_{c}(x, t) \\
& -C(E) N_{c} e^{-\frac{\left(E_{c}-E\right)}{k T}} \rho(x, t, E),
\end{aligned}
$$

где $N_{c}$ - эффективная концентрация проводящих состояний, $C(E)$ - сечение захвата носителей на ловушки с энергией $E$, нормированное на скорость. Первый член в правой части описывает захват, а второй - освобождение носителей с ловушек. После введения обозначений $r(E)=C(E) / C_{0}, C_{0}=1 /\left(\tau_{0} N_{t}\right)=v_{0} / N_{c}$, где $v_{0}$ - частота попыток освобождения, $\tau_{0}$ - время жизни носителей до захвата на ловушки, $N_{t}$ - концентрация ловушек, уравнение баланса приводится к следующему виду:

$$
\begin{aligned}
\frac{1}{r(E)} \frac{\partial \rho(x, t, E)}{\partial t}= & \frac{[g(E)-\rho(x, t, E)] p_{c}(x, t)}{\tau_{0} N_{t}} \\
& -v_{0} e^{-\frac{\left(E_{c}-E\right)}{k T}} \rho(x, t, E) .
\end{aligned}
$$

Уравнение непрерывности, записанное в пренебрежении обычной диффузией (что типично для условий времяпролетного эксперимента),

$$
\frac{\partial p(x, t)}{\partial t}+\mu_{c} F \frac{\partial p_{c}(x, t)}{\partial x}=0
$$

учитывает, что в проводимости участвуют лишь подвижные носители (с подвижностью $\left.\mu_{c}\right), F$ - напряженность электрического поля. В органических материалах, вследствие слабости межмолекулярного взаимодействия, все состояния локализованы. Общепринято, что транспорт носителей является прыжковым, т.е. представляет собой цепочку некоррелированных туннельных перескоков между локализованными состояниями с участием фононов $[1,2]$. В этом случае нет физического различия между
„Проводящими состояниями“ и „ловушками“. Но перенос носителей можно описывать системой уравнений МЗ и в этом случае, поскольку перенос контролируется прыжками с глубоких состояний, на основе концепции транспортного уровня [6,9-13]. Было показано, что прыжки носителей по определенной фракции состояний вносят основной вклад в перенос, так что эти состояния рассматриваются как проводящие, а носители „квазисвободные“. Проводящие состояния находятся в энергетически сравнительно узкой области вокруг определенной энергии (транспортного уровня), которая аналогична краю подвижности, $E_{c}$, а ниже лежащие состояния можно считать ловушками. Характерная частота освобождения носителя с „ловушек“(с энергией $E$ ) практически не зависит от энергии конечного состояния и может быть представлена в виде $v_{0} \exp \left[-\left(E_{c}-E\right) / k T\right]$, аналогично М3 (см. второе слагаемое в правой части уравнения (2)) [10,11]. Ранее $[4,6,7,12]$ коэффициент ПД был найден для предельного случая малой концентрации носителей, т.е. в пренебрежении вторым слагаемым в квадратных скобках в правой части уравнения (2). Далее будет получена зависимость коэффициента ПД от концентрации, с учетом заполнения глубоких ловушек. Разумеется, полученные далее результаты применимы и к неупорядоченным материалам с реальным краем подвижности, разделяющим протяженные и локализованные состояния.

Рассмотрим квазиравновесный режим переноca $[4,7,8,14]$. В нулевом приближении $(\partial \rho / \partial t=0)$ решение уравнения (2) имеет следующий вид [14]:

$$
\rho^{(0)}(x, t, E)=g(E) /\left\lceil 1+\exp \left(\left(E-E_{F}(x, t)\right) / k T\right)\right\rceil,
$$

где квазиуровень Ферми связан с концентрацией квазисвободных носителей:

$$
E_{F}(x, t)=E_{c}-k T \ln \left[\tau_{0} N_{t} v_{0} / p_{c}(x, t)\right] .
$$

В дальнейшем рассмотрено гауссово распределение локализованных состояний по энергии, типичное для органических материалов [1,2]:

$$
g(E)=\frac{N_{t}}{\sqrt{2 \pi \sigma^{2}}} \exp \left(-\frac{E^{2}}{2 \sigma^{2}}\right),
$$

где $\sigma-$ среднеквадратичная вариация. Большинство носителей занимает достаточно глубокие (в типичном случае $\sigma / k T \gg 1)$ состояния с энергиями около $\sigma^{2} / k T$, так что первым слагаемым в уравнении (1) можно пренебречь. Для концентрации квазисвободных носителей было получено [4]

$$
p_{c}(x, t)=\theta p(x, t),
$$

где

$$
\begin{aligned}
\frac{1}{\theta} & =\frac{1}{\tau_{0} \nu_{0}} \int_{-\infty}^{E_{c}} d E g(E) \exp \left(\frac{E_{c}-E}{k T}\right) \\
& \approx \frac{1}{\tau_{0} \nu_{0}} \exp \left(\frac{E_{c}}{k T}+\frac{1}{2}\left(\frac{\sigma}{k T}\right)^{2}\right) .
\end{aligned}
$$


В данном приближении $(\partial \rho / \partial t=0)$ энергетическая зависимость $r(E)$ в уравнении (2) не имеет значения. Для анализа явления полевой диффузии, однако, необходимо учитывать слабую неравновесность, когда левая часть уравнения (2) отлична от нуля, хотя и учитывается по теории возмущений $[4,6]$. Надо заметить, что для вычисления транспортного уровня в данной работе используется широко применяемая [1-3,6-16] модель Миллера-Абрахамса, согласно которой $\omega_{i j}=\omega_{0} \exp \left\lceil-2 \gamma r_{i j}-\left(\left|\Delta E_{i j}\right|+\Delta E_{i j}\right) / 2 k T\right\rceil$, где $\omega_{i j}-$ вероятность перехода носителя в единицу времени на расстояние $r_{i j}$ с состояния энергии $E_{i}$ на состояние с энергией $E_{j}, \Delta E_{i j}=E_{j}-E_{i}, 1 / \gamma-$ радиус локализации волновой функции, $\omega_{0}$ - типичная фононная частота. Поэтому вероятность перехода вниз по энергии (т.е. захвата на ловушку) не зависит от энергии ловушки, так что в вычислениях данной работы, как и ранее $[6,12,16]$, принято $r(E) \equiv 1$. Формально, энергетическую зависимость $r(E)$ нетрудно учесть (см. далее уравнение (10)), однако анализ возможного вида такой зависимости для других моделей темпов переходов (например, модели Маркуса $[17,18]$ ), а также анализ применимости концепции транспортного уровня в этом случае (фактически, эта концепция применена в работе [18]) выходят за рамки данной работы.

Следующее приближение находим, учитывая производную по времени в уравнении (2) в нулевом приближении. Анализ существенно упрощается, если квазиуровень Ферми лежит существенно ниже характерной энергии $-\sigma^{2} / k T$, при этом величина подвижности не зависит от концентрации носителей $[2,15]$, так что можно использовать уравнение (7). Поправка к $\rho^{(0)}$ найдена вычислением $\partial \rho / \partial t$ (см. уравнение $(2))$, в нулевом приближении, с использованием выражений (5), (7):

$$
\begin{gathered}
\rho^{(1)} \approx \rho^{(0)}-\Phi(E) \frac{\partial \rho}{\partial t}, \\
\Phi(E)=\frac{g(E) \theta t^{2}(E)}{\tau_{0} N_{t} r(E)\left(1+\exp \left[-\left(E-E_{F}\right) / k T\right]\right)^{3}} \\
\approx \frac{g(E) \theta t^{2}(E)}{\tau_{0} N_{t} r(E)}, E>E_{F},
\end{gathered}
$$

где $t(E)=v_{0}^{-1} \exp \left[\left(E_{c}-E\right) / k T\right]-$ характерное время освобождения с ,ловушек“ $\left(E \ll E_{c}\right)$. Аргументы функций, кроме энергии, здесь и далее для краткости не пишем. Из уравнения непрерывности нулевого приближения, которое получено из (7) и (3),

$$
\frac{\partial p}{\partial t}=-\mu_{c} \theta F \frac{\partial p}{\partial x}
$$

и из уравнения (9), следует

$$
\rho^{(1)}=\rho^{(0)}+\Phi(E) \mu_{c} \theta F \frac{\partial p}{\partial x} .
$$

Интегрирование уравнения (12) по энергии дает

$$
p_{c}=\theta p-\mu_{c} \theta^{2} \tau F \frac{\partial p}{\partial x}
$$

где $\tau=\int_{-\infty}^{E_{c}} d E \Phi(E)$. Подстановка уравнения (13) в $(9)$ дает уравнение непрерывности с новым диффузионным членом, который зависит от концентрации носителей:

$$
\frac{\partial p}{\partial t}+\mu_{c} \theta F \frac{\partial p}{\partial x}-D_{F}(c) \frac{\partial^{2} p}{\partial x^{2}}=0
$$

где $D_{F}(c)=(\mu F)^{2} \tau(c)-$ коэффициент полевой диффузии, зависящий от относительной концентрации носителей, $c=p / N_{t}, \mu=\mu_{c} \theta-$ квазиравновесная подвижность носителей. Используя (8), заметим, что при условии $E_{F} \ll-\sigma^{2} / k T$ выражение для $D_{F}(c)$ упрощается следующим образом:

$$
\begin{gathered}
D_{F}(c)=\frac{(\mu F)^{2} \theta}{\tau_{0} N_{t}} \int_{-\infty}^{E_{c}} \frac{d E g(E) t^{2}(E)}{\left(1+\exp \left(\frac{E_{F}-E}{k T}\right)\right)^{3}} \\
\approx \frac{(\mu F)^{2} \theta}{\tau_{0}} \int_{E_{F}(c)}^{E_{c}} \frac{d E g(E)}{N_{t}} t^{2}(E), \\
E_{F}(c) \approx E_{c}-k T \ln \left(\tau_{0} v_{0} / \theta c\right) \approx-\frac{1}{2} \frac{\sigma^{2}}{k T}-k T \ln \left(\frac{1}{c}\right), \\
c \leq c_{1}=0.5 \exp \left[-0.5(\sigma / k T)^{2}\right],
\end{gathered}
$$

как следует из (5), (7), (8). Приближенное выражение (15) справедливо при условиях $E_{F} \ll-\sigma^{2} / k T$ и $\sigma / k T \gg 1$, в этом случае интеграл „набирается“ в основном в области $E>E_{F}$, это нетрудно показать как аналитическими оценками приближенных интегралов по энергии (в областях выше и ниже уровня Ферми), так и численными расчетами. В предельном случае $E_{F} \rightarrow-\infty$ (практически $\left.E_{F} \ll-2 \sigma^{2} / k T\right)$ получаем известное $[6,12,16]$ выражение:

$$
\begin{aligned}
D_{F \infty}= & \frac{\sqrt{\pi}}{2} \frac{(\mu F)^{2} \theta}{\tau_{0} v_{0}^{2}} \exp \left(2\left(\frac{\sigma}{k T}\right)^{2}+2 \frac{E_{c}}{k T}\right) \\
& \times\left[1+\operatorname{erf}\left(\frac{1}{\alpha}+E_{c}^{\prime}\right)\right],
\end{aligned}
$$

где $\alpha=k T /(\sqrt{2} \sigma), E_{c}^{\prime}=E_{c} /(\sqrt{2} \sigma), \operatorname{erf}(x)=(2 / \sqrt{\pi})$ $\times \int_{0}^{x} \exp \left(-y^{2}\right) d y-$ функция ошибок. Выражение в квадратных скобках в (15) близко к 2 при $\sigma / k T \gg 1$. Из уравнений (15), (17) следует простое аналитическое выражение:

$$
\begin{aligned}
\frac{D_{F}}{D_{F \infty}}= & \left(\operatorname{erf}\left(\frac{1}{\alpha}+E_{c}^{\prime}\right)-\operatorname{erf}\left(\frac{1}{\alpha}+E_{F}^{\prime}\right)\right) / \\
& \left(1+\operatorname{erf}\left(\frac{1}{\alpha}+E_{c}^{\prime}\right)\right),
\end{aligned}
$$

где $E_{F}^{\prime}=E_{F} /(\sqrt{2} \sigma)=-1 / 4 \alpha+\alpha \ln (c)$, которое и решает задачу о зависимости коэффициента полевой диффузии от концентрации. 


\section{3. Результаты и их обсуждение}

На рисунке представлены зависимости полного коэффициента диффузии, который является суммой коэффициентов полевой и обычной полевой диффузии, $D=\mu k T / e+D_{F}$, от относительной концентрации, полученные вычислением точного и приближенного интеграла в уравнении (15) (см. кривые 1 и 2 соответственно). Точнее, на графике показана нормированная величина $d=\left(D_{0}+D_{F}\right) /\left(D_{0}+D_{F \infty}\right)$, где $D_{0}=\mu k T / e$, которая стремится к единице в предельном случае малой концентрации. Вертикальные штриховые линии отмечают значения концентрации $c_{1}$ и $c_{2}$, при которых $E_{F}\left(c_{1}\right)=-\sigma^{2} / k T$ и $E_{F}\left(c_{2}\right)=-2 \sigma^{2} / k T$. Кроме того, для сравнения, показан результат недавней работы [8]. Надо заметить, что этот результат, будучи более общим, намного сложнее уравнения (16), так как содержит большое число интегралов, которые берутся численно. Очевидно, что приближение в уравнении (15) дает достаточно точный результат при условии $c \ll c_{1}$. С ростом концентрации коэффициент ПД уменьшается, поскольку все больше глубоких состояний полностью заполняются и перестают „работать“ как ловушки, так что разброс времен освобождения носителей с ловушек уменьшается. Результаты данной работы и работы [8] хорошо согласуются в области $c \ll c_{1}$ (при $c>c_{1}$ уравнения (7), (8), (16), (18) не применимы). Коэффициент ПД убывает на 2 порядка в сравнении с предельным значением при малой концентрации, $D_{F \infty}$, при возрастании относительной концентрации до большой величины $c \cong 0.01$ (достигается в полевых транзисторах [19]). Однако даже при такой большой концентрации и даже при рассматриваемом умеренном беспорядке $(\sigma / k T=3)$ коэффициент $D_{F}$ превышает коэффициент обычной диффузии на порядок $\left(D_{f \infty} / D_{0} \cong 10^{3}\right.$ при $\left.F=10^{5} \mathrm{~B} / \mathrm{cm}\right)$.

В нестационарных условиях (например, в условиях времяпролетного эксперимента, если начальное энергетическое распределение носителей близко к квазиравновесному) квазиуровень Ферми, а следовательно, и коэффициент полевой диффузии, зависит от координат и времени (см. уравнение (13)). Рассмотрим зависимость коэффициента ПД от времени вблизи точки с максимальной концентрацией носителей, пренебрегая его зависимостью от координаты, поскольку данная зависимость слаба, а вдали от этой точки мала концентрация носителей. В окрестности рассматриваемой точки приближенное решение уравнения (12) представляет собой гауссовый пакет, вершина которого (точка с максимальной концентрацией) движется с постоянной скоростью, $\mu F t$, а максимальная концентрация убывает со временем по закону

$$
c_{\max }(t)=\frac{\sigma_{0}}{N_{c} \sqrt{4 \pi S(\tau)}}, S(t)=\int_{0}^{t} d t^{\prime} D_{F}\left[c_{\max }\left(t^{\prime}\right)\right],
$$

где $\sigma_{0}-$ начальная поверхностная плотность генерированных носителей (предполагается, что носители

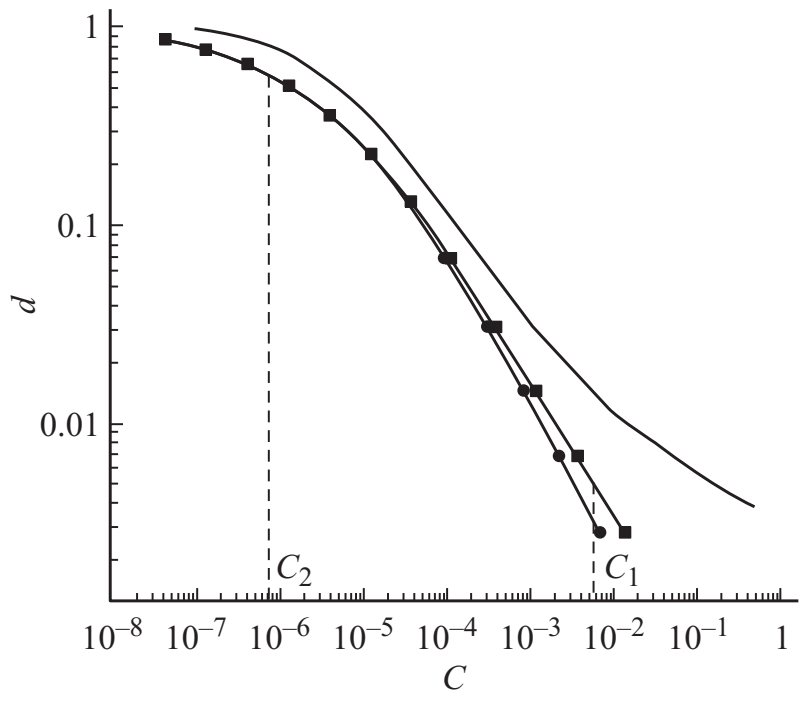

Зависимость нормированного коэффициента диффузии от концентрации для случая $\sigma / k T=3$. Сплошная линия - результат работы [8], линии с кружками и квадратами показывают результаты вычислений согласно точному и приближенному выражениям (15) соответственно.

генерируются в виде тонкого слоя). Исходя из уравнений (19) можно оценить характерные времена $t_{1}$ и $t_{2}$ такие, что $\mu=\mathrm{const}=\mu_{0} \theta$ при $t \gg t_{1}$, и $D_{F} \gg D_{F \infty}$ при $t \ll t_{2}$, т.е. на интервале времени $t_{2} \gg t \gg t_{1}$ концентрация еще достаточно велика, чтобы влиять на коэффициент ПД, но уже достаточно мала, чтобы не оказывать влияния на подвижность. Такая ситуация напоминает „квазидисперсионный“ режим неравновесного транспорта носителей в случае сильно неравновесного начального энергетического распределения генерированных носителей и малой концентрации $[6,12,16]$, когда на широком интервале времени $t_{D} \gg t \gg t_{\mu}$ квазиравновесные значения средней энергии носителей (и подвижности) уже установились, но дисперсия энергетического распределения носителей (и коэффициент ПД) продолжает расти со временем, поскольку квазиравновесная заселенность более глубоких состояний устанавливается позже. Надо заметить, что зависимость коэффициента ПД от времени в квазидисперсионном режиме при низкой концентрации носителей определяется приближенным выражением (15) с заменой квазиуровня Ферми $E_{F}(c)=E_{c}-k T \ln \left(\tau_{0} v_{0} / \theta c\right)$ на демаркационную энергию $E_{d}(t)=E_{c}-k T \ln \left(v_{0} t\right)[6,12,16]$. Характерные времена $t_{\mu}$ и $t_{d}$ определяются из условий $E_{d}\left(t_{\mu, D}\right)=-m \sigma^{2} / k T: t_{\mu, D}=v_{0}^{-1} \exp \left[E_{c} / k T+m(\sigma / k T)^{2}\right]$, где $m=1,2$ в случае $t_{\mu}$ и $t_{D}$ соответственно. Аналогично из условий $E_{F}\left(t_{m}\right)=-m \sigma^{2} / k T(m=1,2)$ следует

$$
\frac{c_{\max }\left(t_{1}\right)}{c_{\max }\left(t_{2}\right)}=\frac{c_{1}}{c_{2}}=\exp \left[-\left(\frac{\sigma}{k T}\right)^{2}\right] \text {. }
$$

Численные расчеты, в согласии с аналитическими оценками, показывают, что в квазидисперсионном режиме 
$D_{F}(t) \cong D_{F \infty}\left(t / t_{D}\right)^{s}$, где $s \leq 1-$ численный коэффициент, который медленно возрастает с ростом параметра беспорядка, $\sigma / k T(s=0.4$ при $\sigma / k T=3$ и $s=0.6$ при $\sigma / k T=4)$ [6]. Аналогично в квазиравновесном режиме при не малой концентрации $D_{F}(c) \cong D_{F \infty}\left(t / t_{2}\right)^{s}$. Дифференцируя уравнение (19) по времени и решая получившееся дифференциальное уравнение с использованием приближенной степенной зависимости $D_{F}(c)$ и уравнения (20), нетрудно получить:

$$
\begin{gathered}
c_{\max }(t)=c_{2}\left(t / t_{2}\right)^{-1 /(2-s)}, \quad D_{F}(t)=D_{F \infty}\left(t / t_{2}\right)^{s /(2-s)}, \\
t_{2}=\frac{1}{2 \pi(2-s) D_{F \infty}}\left(\frac{\sigma_{0} \theta t_{D}}{N_{t} \tau_{0}}\right)^{2} \\
t_{1}=t_{2} \exp \left[-(2-s)\left(\frac{\sigma}{k T}\right)^{2}\right]
\end{gathered}
$$

Очевидно, характерные времена $t_{1}$ и $t_{2}$ убывают с убыванием начальной поверхностной плотности $\sigma_{0}$. Полагая $\sigma_{0}=\xi \varepsilon \varepsilon_{0} F / e$, где фактор $\xi \ll 1$ согласно условиям времяпролетного эксперимента, $\varepsilon \approx 3$ - относительная диэлектрическая проницаемость, $\varepsilon_{0}-$ электрическая постоянная, получаем следующие оценки:

$$
\begin{gathered}
\frac{t_{2}}{t_{D}}=\frac{\xi^{2}}{30}\left(r_{c} N_{t}^{1 / 3}\right)^{-2} \exp \left[\frac{1}{2}\left(\frac{\sigma}{k T}\right)^{2}\right], \\
\frac{t_{1}}{t_{\mu}}=\frac{\xi^{2}}{30}\left(r_{c} N_{t}^{1 / 3}\right)^{-2} \exp \left[\left(s-\frac{1}{2}\right)\left(\frac{\sigma}{k t}\right)^{2}\right],
\end{gathered}
$$

где $r_{c}=e^{2} / 4 \pi \varepsilon \varepsilon_{0} k T-$ кулоновский радиус. Полагая $\xi=0.1$, при комнатной температуре и $\sigma / k T=4$ получаем $t_{2} / t_{D} \cong 0.01, t_{1} / t_{\mu} \cong 10^{-5}$. Таким образом, постоянная подвижность устанавливается (вследствие падения концентрации) очень быстро, в то время как коэффициент ПД возрастает на длительном временно́м интервале даже при достаточно малой начальной концентрации носителей заряда, типичной для времяпролетного эксперимента, при этом рост происходит медленнее, чем в случае сильно неравновесной начальной генерации $[6,12,16]$ (см. уравнение (21)).

\section{4. Заключение}

В данной работе получено уравнение непрерывности, которое описывает дрейф и диффузию носителей заряда в случае, когда концентрация носителей достаточно велика, чтобы вызывать значительное уменьшение коэффициента полевой диффузии, но достаточно мала, чтобы не влиять на подвижность и напряженность электрического поля в слое исследуемого материала. Получено простое аналитическое выражение для зависимости коэффициента полевой диффузии от концентрации носителей заряда, достаточно точное в практически значимом диапазоне концентраций. Проведен анализ зависимости коэффициента полевой диффузии от времени для квазиравновесного режима переноса в нестационарных условиях, когда концентрация зависит от времени (например, в условиях времяпролетного эксперимента в том случае, когда энергия возбуждающего излучения не достаточна для того, чтобы начальное энергетическое распределение носителей было сильно неравновесным). Показано, что и в случае квазиравновесной начальной генерации носителей коэффициент полевой диффузии на длительном интервале времени возрастает по степенному закону (при постоянной подвижности), хотя и с другим показателем, чем в случае сильно неравновесной начальной генерации и низкой концентрации носителей $[6,12,16]$. Это обстоятельство надо учитывать при анализе данных времяпролетных экспериментов.

Эта работа поддержана программой повышения конкурентоспособности НИЯУ МИФИ).

\section{Список литературы}

[1] H. Bässler. Phys. Status Solidi B, 175, 15 (1993).

[2] S. Baranovskii. Phys. Status Solidi B, 251, 487 (2014).

[3] P.M. Borsenberger, R. Richert, H. Bässler. Phys. Rev. B, 47, 4289 (1993).

[4] A.I. Rudenko, V.I. Arkhipov. Phil. Mag. B, 45, 177 (1982).

[5] A. Hirao, H. Nishizawa. Phys. Rev. B, 56, R2904 (1997).

[6] V.R. Nikitenko, H. von Seggern, H. Bässler. J. Phys.: Condens. Matter, 19, 136210 (2007).

[7] A.V. Nenashev, F. Jansson, S.D. Baranovskii, R. Österbacka, A.V. Dvurechenskii, F. Gebhard. Phys. Rev. B, 81, 115204 (2010).

[8] Ling Li, Nianduan Lu, Ming Liu, H. Bässler. Phys. Rev. B, 90, 214107 (2014).

[9] S.D. Baranovskii, I.P. Zvyagin, H. Cordes, S. Yamasaki, P. Thomas. Phys. Status Solidi B, 230, 281 (2002).

[10] V.I. Arkhipov, E.V. Emelianova, G.J. Adriaenssens. Phys. Rev. B, 64, 125125 (2001).

[11] V.R. Nikitenko, M.N. Strikhanov. J. Appl. Phys., 115, 073704 (2014).

[12] В.Р. Никитенко, А.П. Тютнев. ФТП, 41 (9), 1118 (2007).

[13] И.П. Звягин. Кинетические явления в неупорядоченных полупроводниках (М., МГУ 1984).

[14] V.I. Arkhipov, P. Heremans, E.V. Emelianova, G.J. Adriaenssens. Appl. Phys. Lett., 79, 4154 (2001).

[15] R. Coehoorn, W.F. Pasveer, P.A. Bobbert, M.A.J. Michels. Phys. Rev. B, 72, 155206 (2005).

[16] V.R. Nikitenko, H. von Seggern. J. Appl. Phys., 102, 103708 (2007).

[17] R.A. Marcus. Rev. Mod. Phys., 65, 599 (1993).

[18] J. Cottaar, R. Coehoorn, P.A. Bobbert. Phys. Rev. B, 85, 245205 (2012).

[19] C. Tanase, P.W.M. Blom, D.M. de Leeuw, E.J. Meijer. Phys. Status Solidi A, 201, 1236 (2004).

Редактор Г.А. Оганесян 


\section{Field-stimulated diffusion in disordered organic materials under conditions of filling of deep states}

\section{V.R. Nikitenko, A.Yu. Kudrov}

National Research Nuclear University MEPhl

(Moscow Engineering Physics Institute),

115409 Moscow, Russia

Abstract A simple analytic model of field-stimulated diffusion coefficient is developed in this work at moderate concentration of charge carriers. Hopping transport is described by the multiple trapping model on the base of transport level concept. Continuity equation with concentration-dependent diffusion coefficient is obtained and the time dependence of field-stimulated diffusion coefficient is founded under non-stationary conditions. Estimations of time intervals are obtained, at which mobility and diffusion coefficient are affected by filling of deep states under the conditions of time-of-flight experiment. Increasing of field-stimulated diffusion coefficient during long time interval is shown, although mobility is constant, reminiscent to the case of non-equilibrium initial generation of carriers in low-concentration limit. 